\title{
Design of Sub-THz Traveling Wave Tubes for High Data Rate Long Range Wireless Links
}

\author{
Rupa Basu, Laxma R. Billa, Rosa Letizia and Claudio Paoloni \\ Engineering Department, Lancaster University, Lancaster, United Kingdom \\ E-mail: c.paoloni@lancaster.ac.uk
}

Received xxxxxx

Accepted for publication $\mathrm{xxxxxx}$

Published xxxxxx

\begin{abstract}
The development of high capacity wireless networks, to satisfy the increasing demand of mobile high-speed internet, is stimulating the exploitation of the wide frequency bands in the millimeter and $\mathrm{THz}$ range. Above $100 \mathrm{GHz}$, the high atmosphere attenuation is the major obstacle for wireless links of adequate length, due to the low power available from solid state amplifiers. Traveling wave tubes are promising devices capable to generate multi-watt power at sub-THz frequency. In this paper, the design and performance of a Traveling Wave Tube in the $210-250 \mathrm{GHz}$ band with about $1 \mathrm{~W}$ output power will be described, to power a 40 Gigabits per second optical transmitter for $1 \mathrm{~km}$ point-to-point link. A novel approach for a simple and low-cost fabrication, based on the use of the double corrugated waveguide is proposed.
\end{abstract}

Keywords: Vacuum electronics, traveling wave tubes, wireless communications, double corrugated waveguide, 5G

\section{Introduction}

Many applications at millimeter waves and $\mathrm{THz}$ frequencies are not fully exploited due to the lack of compact amplifiers with power level in the Watt or multi-Watt range. In most of the cases, the only options are bulky devices, such as Far Infrared (FIR) lasers (e.g. for plasma diagnostic in nuclear fusion) or long multiplication chains (e.g. for imaging or millimeter wave radars).

An area of great interest for the use of sub-THz frequencies is high speed wireless networks for supporting the growing demand of internet traffic. The wide frequency bands available above $100 \mathrm{GHz}$ could permit data rate at level of tens of Gigabits per second $(\mathrm{Gb} / \mathrm{s})$ if adequate transmission power would be available $[1-6]$. G-band (in the range $200-260$ $\mathrm{GHz}$ ) is of particular interest with about $60 \mathrm{GHz}$ of useful band characterized by relatively low atmosphere attenuation. High data rate has been already demonstrated at laboratory level by photonic devices such as unitravelling-carrier photodiodes (UTC-PD) [3]. A UTC-PD transmitter provided about $1 \mathrm{~mW}$ in the range $200-260 \mathrm{GHz}$, with data rate exceeding $30 \mathrm{~Gb} / \mathrm{s}$, in laboratory environment. However, the low transmission power of solid-state transmitters prevents the use of these frequencies in real environment due to the high atmosphere attenuation, especially in case of rain. This limits the transmission range to a few tens of meters. A point-topoint sub-THz link should be hundreds of meters long to be attractive to users. The use of high gain antennas (e.g. up to 55 $\mathrm{dBi}$ ) can compensate the low transmission power permitting to achieve long transmission range, but it brings substantial alignment difficulties. A simple link budget calculation, including rain attenuation, based on the use of moderate high gain antennas (30 - $35 \mathrm{dBi}$ ) for an easy alignment, shows that at least $0.3 \mathrm{~W}$, with $1 \mathrm{~W}$ target, are needed above $200 \mathrm{GHz}$ for a range of about $1 \mathrm{~km}$. Presently, no solid-state amplifier is able to produce this level of power. Even if solid-state technology is progressing fast, it is far from providing Watt level above $100 \mathrm{GHz}$. GaN power amplifiers have demonstrated about $2 \mathrm{~W}$ up to $100 \mathrm{GHz}$. However, at the increase of frequency, the output power from solid state devices decreases sharply, e.g. $10 \mathrm{~mW}$ at $300 \mathrm{GHz}$ [7].

Vacuum electronic devices, such as Traveling Wave Tubes (TWT), are the most promising, compact and affordable 
devices for producing high power at millimeter waves and sub-THz frequencies $(80-1100 \mathrm{GHz})$ [4].

The working mechanism of Traveling Wave Tubes (Fig.1) is based on the transfer of energy from of an electron beam to the radiofrequency (RF) field propagating in a proper metal waveguide, called interaction structure or slow wave structure (SWS), in high vacuum $\left(10^{-7}-10^{-8}\right.$ Torr). A TWT consists of an electron gun to generate the electron beam, the slow wave structure (SWS) where part of the energy of the electrons is transferred to the signal, the collector to collect the electrons at the end of the SWS, the magnetic focusing system to confine the electron beam inside the SWS and the RF window to vacuum sealing the flanges. When the velocity of electrons is slightly higher than the phase velocity of the wave, the current modulation of the beam produces high density electron bunches in correspondence of the retarded fronts of the wave. The amplification of the RF field is obtained by the transfer of part of the kinetic energy lost by the bunches when retarded by the wave front. The electron beam is confined in a cylinder or rectangular section geometry along the longitudinal dimension of the TWT by an axial magnetic focusing field, to avoid the collision of electrons with the metal walls and consequent loss of energy. The reason of the better performance of TWTs at sub-THz frequencies in comparison to solid-state devices is the collisionless flowing of electrons in vacuum without any loss of energy as in atomic reticules.

In recent years, a great effort has been devoted to design and build compact TWTs up $1 \mathrm{THz}$. The technology is still at the state of the art and only a few prototypes have been reported in literature [ $8-10]$. Two main challenges have to be addressed. One is the low interaction properties of SWSs above the microwave frequencies range with the need of topologies suitable for microfabrication. The double corrugated waveguide (DCW) will be considered in the following. The DCW is a simple slow wave structure, made of two parallel rows of pillars enclosed in a rectangular waveguide [16] suitable for sub-THz TWTs. The DCW was extensively studied for G-band applications, with a focus on the saturation behavior [17].

The second challenge is the fabrication of the interaction structure. The dimensions of the parts are a fraction of the wavelength, that at sub-THz frequencies is a few millimeters. TWTs are a combination of three-dimensional metal parts that require high precision assembly. In addition, the decreasing skin depth at the increase of the frequency requires a highquality surface finishing to provide surface roughness below $100 \mathrm{~nm}$, to keep the ohmic losses close to theoretical values. The introduction of advanced fabrication technologies, such as high precision CNC milling [11-13] or photolithographic processes (LIGA, acronym of Lithography, Electroplating, and Molding) [14-15], has permitted to produce high precision metal structures with the required dimensions and high quality metal surfaces.
The opportunity to have high power traveling wave tubes up to the sub-THz range has opened the route to the design of new high data rate front ends for long links at millimeter waves and sub-THz frequency [6].

In this paper, on the basis of the results presented in [17], the design of a novel traveling wave tube amplifier, based on the double corrugated waveguide, in the $210-250 \mathrm{GHz}$ frequency band is reported. The novelty of the design lies in use of only one section of SWS to provide $30 \mathrm{~dB}$ gain and high linearity over about $40 \mathrm{GHz}$ bandwidth. The output power of $1-\mathrm{W}$ is suitable to enable a high capacity link longer than $1 \mathrm{~km}$, fed by a UTC-PD transmitter, to deliver $40 \mathrm{~Gb} / \mathrm{s}$ data rate by using ASK modulation, for an unprecedented link capacity.

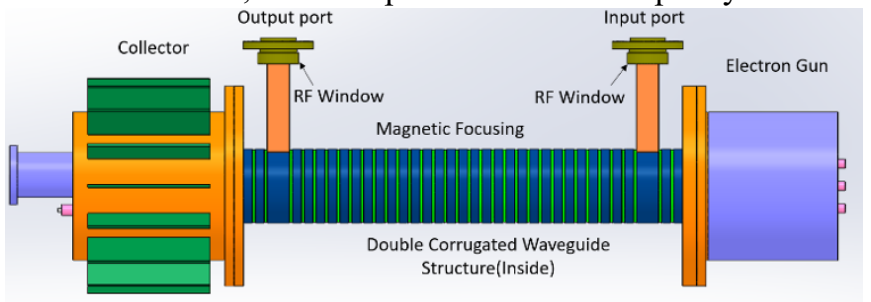

Fig. 1 G-band traveling wave tube rendering

The paper includes two sections. Section 2 describes the design approach for the required dispersion, interaction properties and S-parameters of the DCW structure. Section 3 discusses the large signal performance of the DCW TWT.

\section{TWT design and cold parameters}

A typical microwave TWT has gain in the range of $40 \mathrm{~dB}$ with tens or hundreds of Watt of output power. This high gain poses a risk of oscillations. To reduce this risk, the SWS is commonly divided in two sections with lower gain (about 20 $\mathrm{dB}$ each), electromagnetically isolated by a sever. The sever is a short section of a waveguide under cutoff that permits the transit of the electron beam only. In this way, the risk of oscillations due to high level of reflections from the imperfect matching inside the SWS is very low. The assembly of two sections of SWS and the sever is quite complex for fabrication and assembly when the dimensions are in the sub-millimeter range, to support sub-THz wavelengths. This makes the tube fabrication expensive and time consuming. To note that the cost of components is a key parameter for the wireless application market. The use of a single section SWS would substantially simplify the assembly with significant cost benefit. The design approach of the proposed G-band TWT assumes that above $100 \mathrm{GHz}$, given the high intrinsic losses and the relatively low power, is permitted to consider satisfied the gain-stability condition as it will be demonstrated in the following. This would permit to have only one section SWS with a higher gain than allowed at microwaves. 


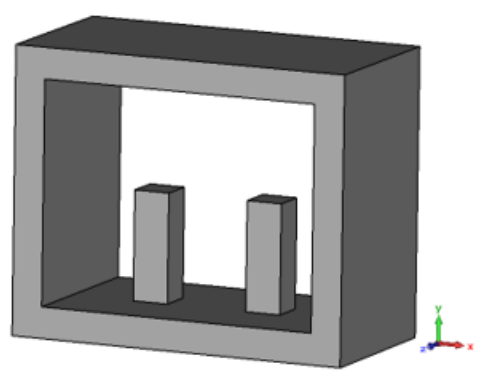

Fig. 2 Double corrugated waveguide unit period

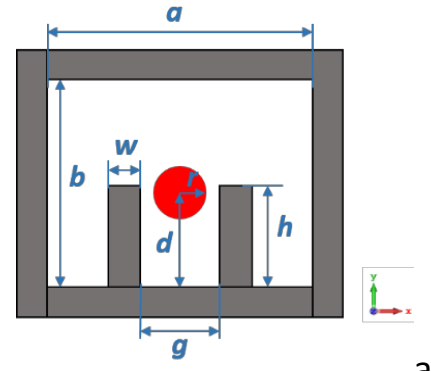

a)

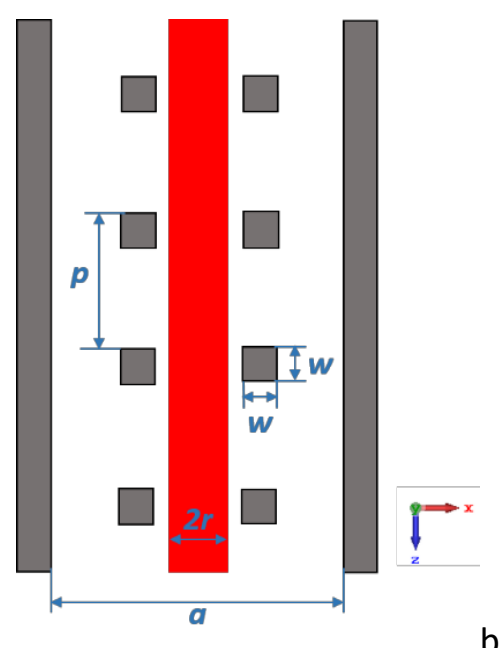

b)

Fig. 3 (a) Front and (b) top view schematic of the DCW. The red shape is the electron beam.

In this paper, a TWT based on one SWS section with about $30 \mathrm{~dB}$ gain and the design and performance of its RF components is discussed. The electron gun, the magnetic focusing system and the collector are not in the scope of the paper, but these parts are designed on the basis of the TWT specifications.

The slow wave structure has to provide the best interaction between the electron beam and the RF signal. The RF window that has to assure the highest transparency to the RF signal at the ports. The design and simulation of all the parts require three dimensional electromagnetic simulations. The double corrugated waveguide (Fig.2) is a simple structure suitable for microfabrication by both photolithographic with electroforming and $\mathrm{CNC}$ milling. It was already built and measured at different frequencies up to $1 \mathrm{THz}$ with very good results [11], [18], [19]. This permits to assume the numerical simulation are reliable without the need of further experimental validation.

\subsection{Dispersion and Interaction impedance}

The design of the DCW is based on the definition of the dimensions of the unit cell (Fig.2), computed by the Eigenmode solver of CST, to achieve the useful bandwidth with the correct dispersion. A compromise between the dimensions and the beam voltage is needed. The voltage of the electron beam is set at $12.3 \mathrm{kV}$. This value is a compromise between the best focusing, the power supply cost and the energy of the electrons for high efficiency.

The first harmonic of the fundamental mode $(2<\phi / \pi<3)$ is chosen to operate with a low beam voltage [17].

Table I - DCW dimensions

\begin{tabular}{|l|l|}
\hline Parameters & Dimensions $(\boldsymbol{\mu m})$ \\
\hline SWS width (a) & 550 \\
\hline SWS height (b) & 430 \\
\hline SWS period (p) & 345 \\
\hline Tunnel width (g) & $160-200$ \\
\hline Pillar height (h) & 210 \\
\hline Pillar width (w) & 70 \\
\hline Pillar thickness (w) & 70 \\
\hline Beam radius ( $r$ ) & 70 \\
\hline Beam center (d) & 180 \\
\hline
\end{tabular}

In Fig.3 the schematic of the DCW is shown. The dimensions listed in Table I provide a dispersion curve matching the electron velocity in the $210-250 \mathrm{GHz}$ band.

The dispersion curve with the superimposed beam line, defined as the locus of the electron velocity in the dispersion plane, is shown in Fig. 4. The beam line is computed as:

$$
f=\frac{\varphi v_{e}}{2 \pi p}
$$


where $\varphi$ is the propagation phase of the considered space harmonic, $p$ is the period, $v_{e}$ is the electron velocity computed as:

$$
v_{e}=\sqrt{2 \mathrm{Ve} / \mathrm{m}}
$$

where $V$ is the beam voltage, $e$ is the electron charge and $m$ is the mass of the electron.

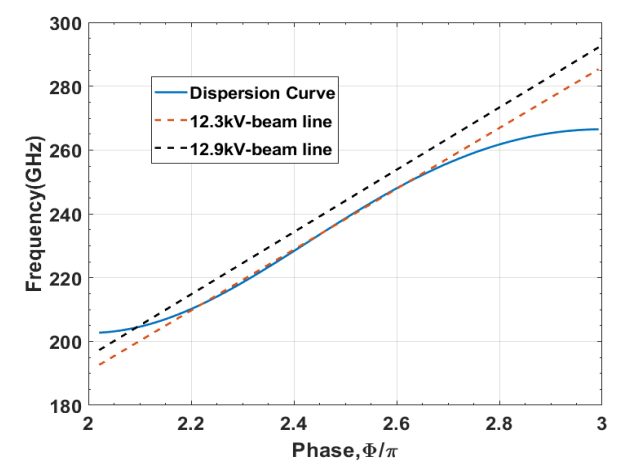

Fig. 4 Dispersion curve of the DCW with the beam lines (initial and from PIC simulations).

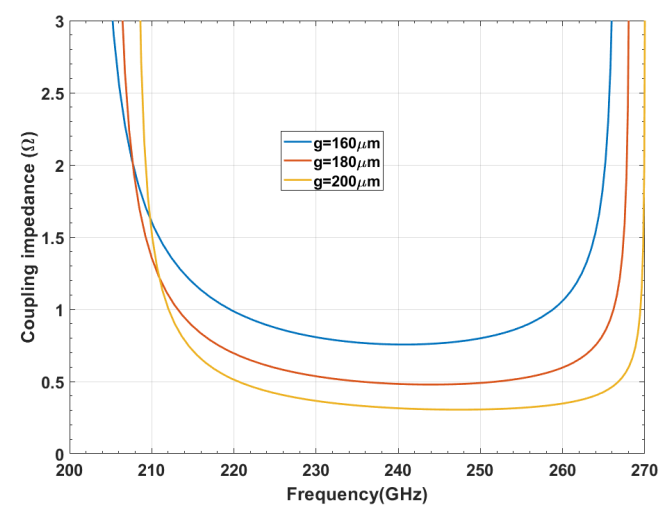

a)

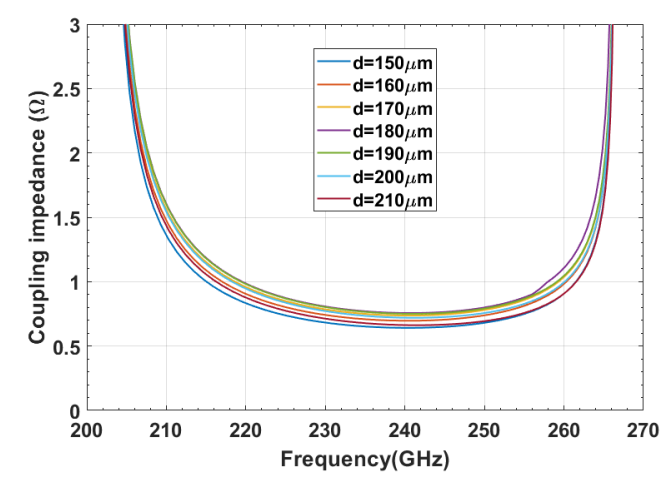

b)

Fig. 5. Coupling impedance as a function a) of the beam tunnel width and $b$ ) vertical position $(g=$ $160 \mu \mathrm{m})$.

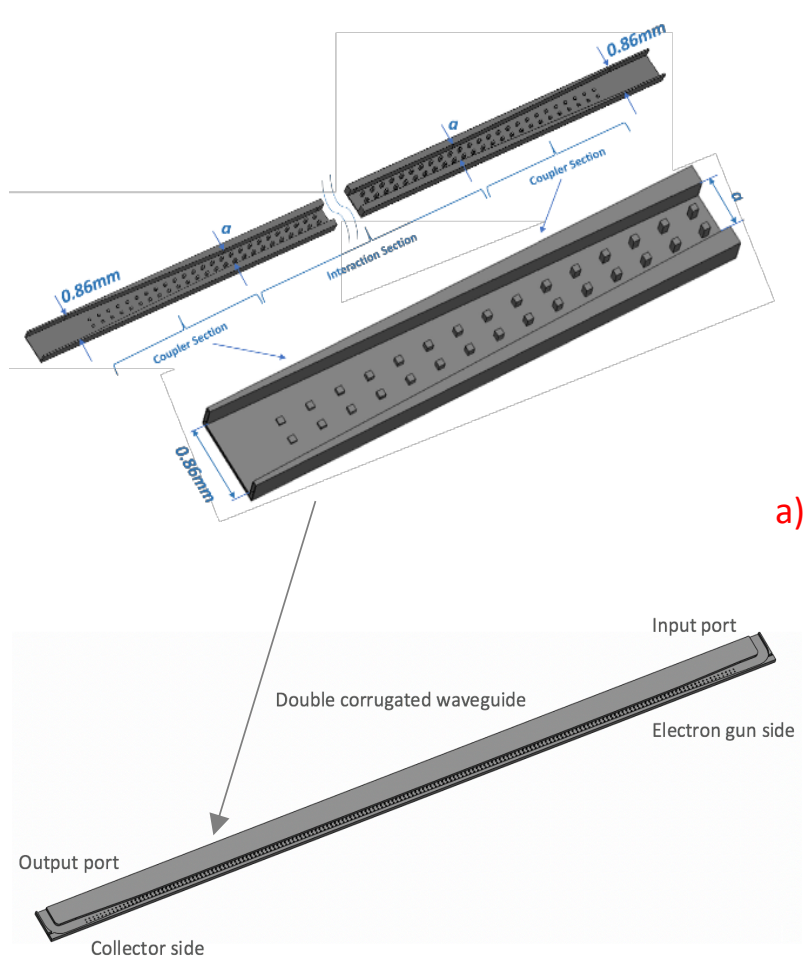

b)

Fig. 6 Details of a) the Input and output couplers and b) the full structure.

The overlap of the dispersion curve and beam line over the full band in Fig. 4 demonstrates the good synchronism between the electron velocity and the wave. About $17 \%$ bandwidth, in the $210-250 \mathrm{GHz}$ range, centered at $230 \mathrm{GHz}$ is obtained.

The coupling or interaction impedance is the other important parameter to characterize the properties of a SWS. It defines the amount of energy transfer from the electron beam to the RF fields and strongly depends on the width of the beam channel, i.e. the distance $g$ between the two rows of pillars. The width $g$ depends on the diameter of the electron beam, to assure it has the required distance from the metal walls to avoid collisions with the electrons and possible overheating and loss of energy. Due to the electric field profile, more intense closer to the metal walls of the pillars, the increase of $g$ reduces the electric field at the center of the beam tunnel and consequently reduces the interaction impedance. The electron gun is designed to produce a circular cross-section beam with radius of $70 \mu \mathrm{m}$. The interaction impedance of the DCW SWS is computed for three different values of the beam tunnel, $g=160,180$ and $200 \mu \mathrm{m}$, corresponding to 10,20 and $30 \mu \mathrm{m}$ gap with the metallic walls of the pillars (Fig.5a). The coupling impedance over the band increases at the decrease of $g$. A gap of $20 \mu \mathrm{m}$ between the internal surface of the pillars and the beam outer radius is 
considered safe, however, due to the expected low beam modulation that permits to fully confine the beam, $10 \mu \mathrm{m}$ gap is acceptable and assure better performance. A study on the vertical position of the beam has been performed showing that an accurate choice of position improves the coupling impedance (Fig. 5b).

\subsection{Input and output couplers}

Next, the design of the full RF structure including a number of periods similar to the final TWT and of the output and input couplers is performed (Fig. 6b). The Scattering parameters of the full SWS are simulated by the time domain solver in CST to verify the quality of the signal transmission. The couplers must assure the transition from the hybrid mode propagating in the DCW to the $\mathrm{TE}_{10}$ mode at the flanges (WR-03 standard waveguide flange dimensions, $0.860 \times 0.430 \mathrm{~mm}$ ).

An effective topology for the input and output couplers for a DCW SWS is shown in Fig. 6a [17]. The coupler consists of a number of periods with linear decreasing tapering of the height $h$ of pillars towards the flanges. To improve the coupling performance, a tapering of the width $a$ of the waveguide was added. 15 periods of tapered pillars for the input coupler and the output coupler were used, with the heights optimized for the best matching [17]. A particular effort has been devoted to achieve the best reflection coefficients to assure high stability of the TWT. The fabrication will be performed by high accuracy CNC milling, that provides surface roughness below the skin depth of copper $(130 \mathrm{~nm}$ at $220 \mathrm{GHz})$. A reduced conductivity of copper $\left(\sigma=3.5 \times 10^{7} \mathrm{~S} / \mathrm{m}\right)$ is used in the simulations to consider imperfections in surface roughness. The S-parameters for the DCW with 160 periods and two couplers with 15 periods each are shown in Fig.7.

The reflection coefficient $\left(\mathrm{S}_{11}\right)$ of the full structure is below $-17 \mathrm{~dB}$ over the operating frequency band, showing improvement over the $-12 \mathrm{~dB}$ in [17]. The transmission losses

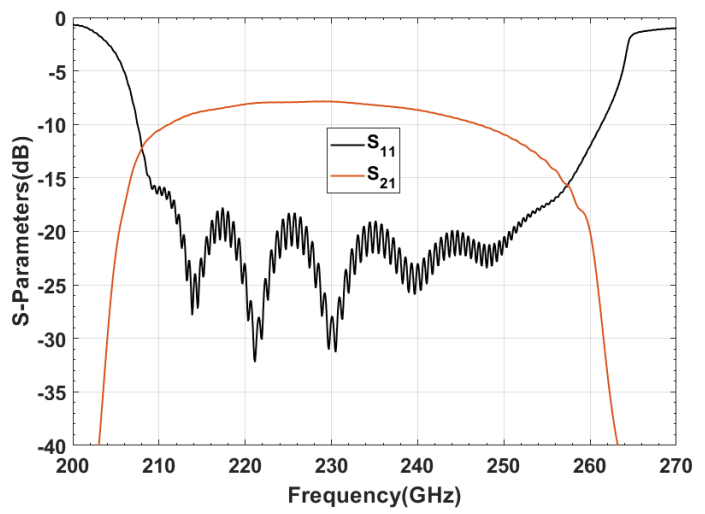

Fig. 7 S-parameters of the 160 period DCW and couplers.
$\left(\mathrm{S}_{21}\right)$ are in the range $-8 /-10 \mathrm{~dB}$ over the frequency range. High losses are expected due to the sub-THz frequency range.

\subsection{RF window}

The RF window is a disk of material transparent to the waves but able to maintain high vacuum in the TWT. It is usually placed close to the flanges. The main challenge is to minimize the losses for a full transmission of the signals. The structure here proposed is very simple, to easy the fabrication. The window is made of an Alumina disc $\left(\varepsilon_{\mathrm{r}}=9.6\right)$ brazed between two cylindrical waveguides that form a pillow box cavity. The cavity is connected to the WR3 waveguide sections on both sides. The design and simulation, performed by CST, demonstrate very good transmission over the full frequency band (Fig.8).

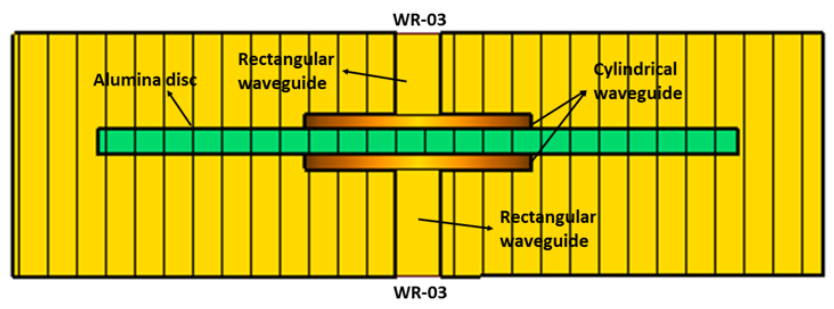

(a)

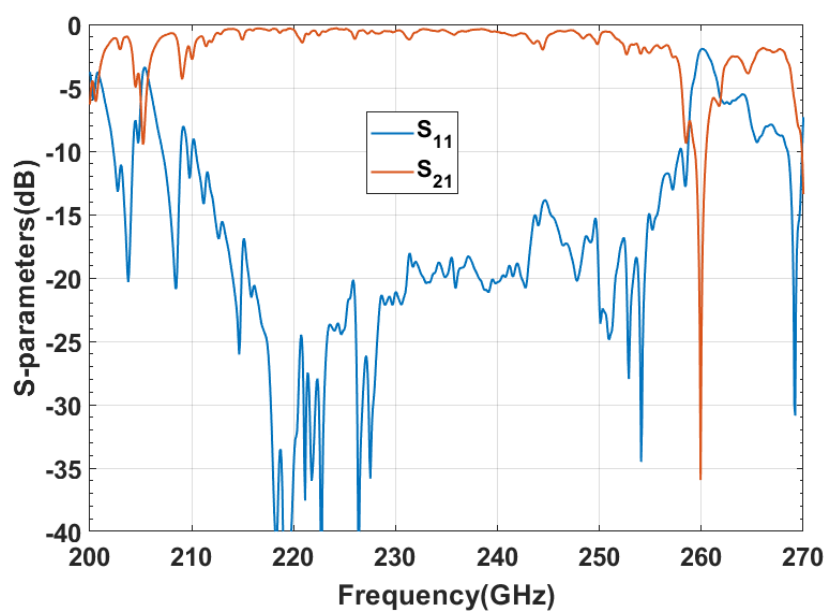

(b)

Fig. 8 Pillbox RF window a) Schematic b) S-parameters.

\section{TWT large signal analysis}

The simulation of the cold parameters described in the previous section gives the first estimate of the potential performance of the TWT in terms of bandwidth and interaction of the beam with the RF field. The simulations of 
large signal behavior to derive the output power are much more complex. They are performed by three-dimensional particle in cells (PIC) simulators that model the interaction of the electron in the electron beam with the electromagnetic field in the SWS. PIC simulations are very time consuming due to the high number of mesh cells required to model the SWS and the number particles, in the order of millions to model the electrons in the beam, for numerical accuracy. The simulations were performed by CST-Particle Studio [22] (with the aid of NVIDIA P100 GPU that permits to reduce of a factor 10 the simulation time).

The simulation domain is the same of Fig. $6 \mathrm{~b}$ with the addition of the electron beam traveling along the two rows of pillars. WR03 flanges are used for the input and output port. The $12.3 \mathrm{kV}$ beam voltage defined in Fig. 4 is of reference to estimate the bandwidth. The optimum beam voltage of 12.9 $\mathrm{kV}$ to provide the highest output power is found after a number of iterations by PIC simulations. This value assures the best difference between the phase velocity of the wave and the electron velocity for maximizing the interaction. The beam current is set at $50 \mathrm{~mA}$.

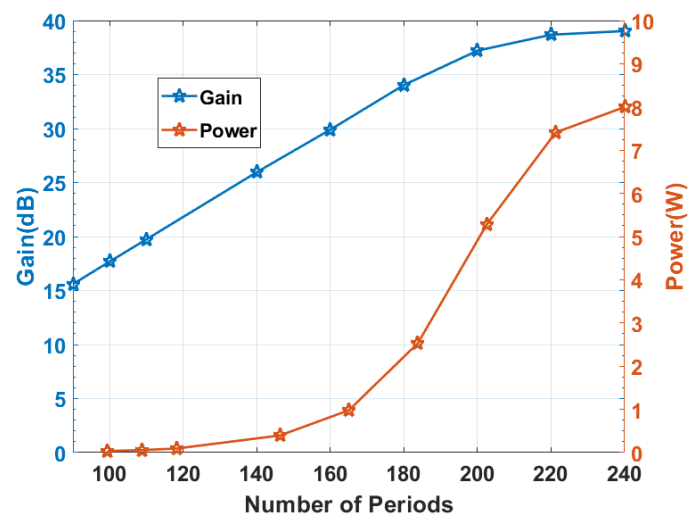

Fig. 9 Gain and output power as a function of the number of periods with $1 \mathrm{~mW}$ input power.

The focusing magnetic field is $0.6 \mathrm{~T}$. An input power of $1 \mathrm{~mW}$ is assumed. The design target is $30 \mathrm{~dB}$ gain and $1 \mathrm{~W}$ output power in the $210-250 \mathrm{GHz}$ band.

The first step is to define the number of periods of the DCW needed to provide the required gain and output power. Fig.9 shows the gain and the output power as a function of the number of periods computed at a single frequency $(240 \mathrm{GHz})$ and $1 \mathrm{~mW}$ input power. At least 130 periods are needed to have $25 \mathrm{~dB}$ gain, corresponding to $0.3 \mathrm{~W}$, which can be achieved in a single section without any oscillations. At least 160 period are needed for $1 \mathrm{~W}$.

The performance of two TWTs with different number of periods, 140 and 160 periods respectively, are discussed.

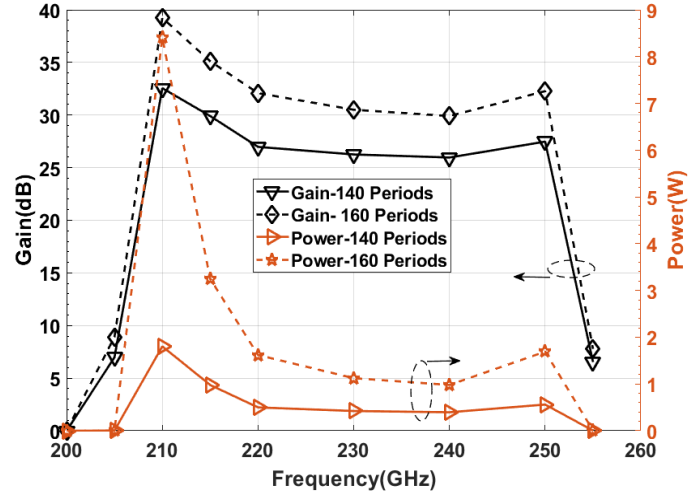

Fig. 10 Output power and gain as a function of input power for 140 periods and 160 periods.

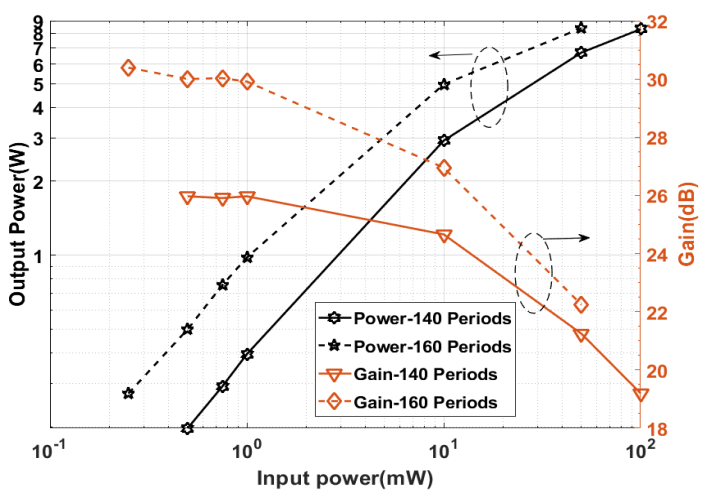

Fig. 11 Output power and gain as a function of input power for 140 periods and 160 periods (computed at $240 \mathrm{GHz}$ ).

The output power and gain as function of frequency are shown in Fig. 10 for the two cases. The DCW with 140 periods provides about $0.5 \mathrm{~W}$ output power over the bandwidth. About $1 \mathrm{~W}$ is achieved by a single section TWT with 160 periods. In principle, increasing the number of periods, the gain increases, but also the risk of oscillations increases. However, in both the cases the simulations do not show presence of oscillations. This is due, as previously discussed, to the ohmic losses higher than conventional microwave TWTs that strongly attenuate the reflected backward propagating signal. In addition, the low interaction impedance, typical of the millimeter wave interaction structures, also determines a low efficiency in the transfer of the energy from the beam to the RF field.

As a verification, the gain-stability equation is applied [23]

$$
G-S_{21}-S_{11}-S_{22}>0
$$

where $G$ is the gain of the TWT, and $S \boldsymbol{x} \boldsymbol{x}$ are the Sparameters of the interaction structure (unit is $d B$ ). The 
equation applied to the 160 period DCW provides a stability margin of more than $10 \mathrm{~dB}$ that indicates a stable behavior.

Figure 10 also shows that the bandwidth is about $40 \mathrm{GHz}$ $(17 \%)$ as predicted by the dispersion curve in Fig. 4. The linearity of the TWT, computed at $240 \mathrm{GHz}$, is shown Fig. 11 where the variation of output power against the value of input power is reported. In principle, this linearity permits the use of a high modulation scheme (up to 16QAM for short distance). The front end will use ASK that has low signal to noise ratio requirements and is sufficient for $40 \mathrm{~Gb} / \mathrm{s}$ in the given band.

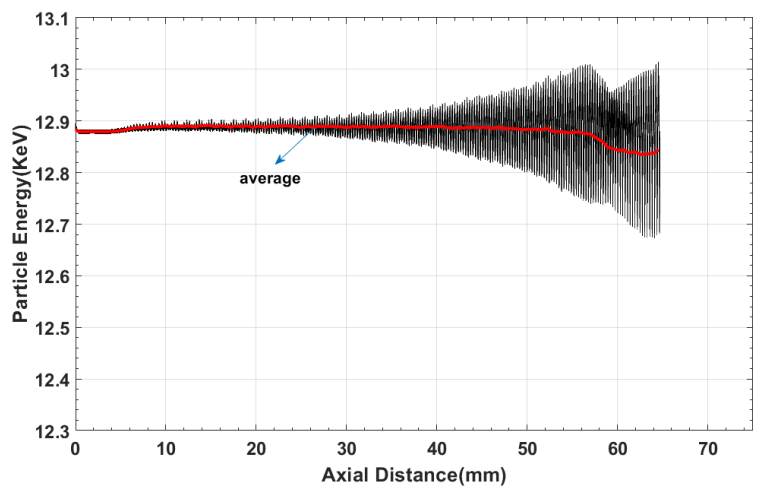

(a)

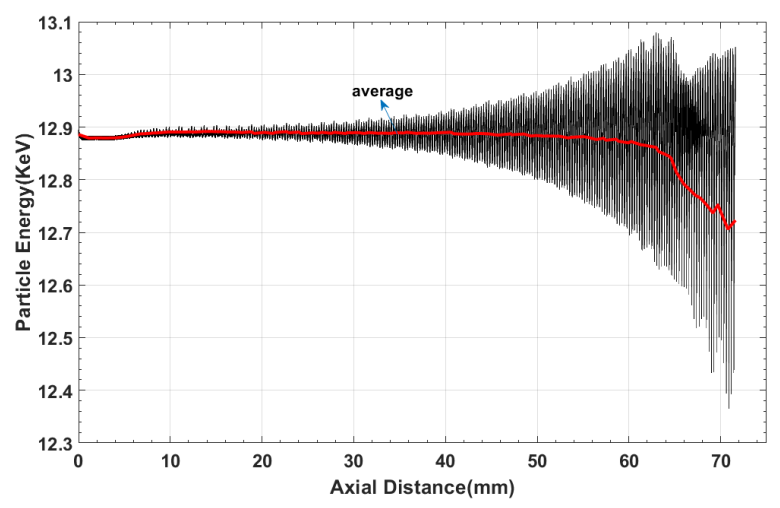

(b)

Fig.12 Electron energy along the TWT longitudinal axis for a) 140 and b) 160 periods. The superimposed red line is the average energy. (computed at 240 $\mathrm{GHz}$ ).

The electron energy as a function of the axial distance along the length of the TWT shows the variation of the energy of electrons due to the interaction with the RF field (Fig.12). The average energy (red line) measures the amount of energy lost by the beam. Part of this lost energy is transferred to the RF field. It is noteworthy that the energy lost by the beam is higher at the end of the 160 period DCW than the 140 period DCW. This explains the higher power of the 160 period DCW.
The increased depth of the modulation informs that the behavior is closer to saturation as in Fig. 9, but still in linear region.

Figure 13 shows the visualization of the strengthening of the bunching of the electron beam in different sections of the DCW. It is notable the high level of bunching at the end of the DCW as expected.

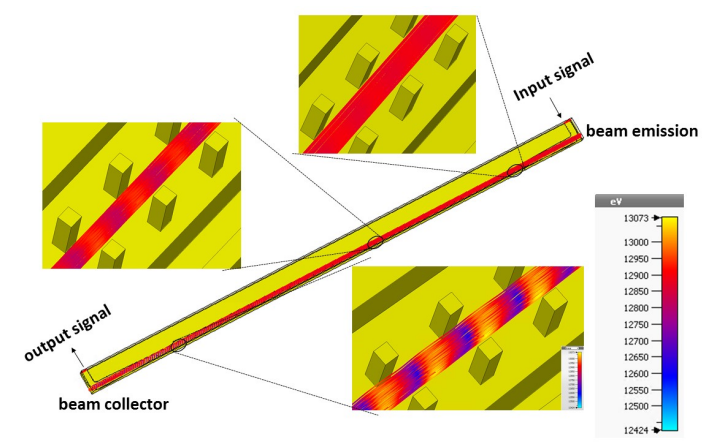

Fig. 13 Beam sections with different level of bunching.

\section{Conclusions}

The design and performance of a $210-250 \mathrm{GHz}$ Traveling Wave Tube based on the double corrugated waveguide have been discussed. In comparison to solid state amplifiers, the G-band TWT offer higher power in a wide frequency band. The high losses and low power at sub-THz frequency permits to achieve higher gain than at microwaves with a single SWS sections, thus reducing the structure complexity. The easier fabrication makes the presented TWT a promising device for large scale production for long range, high data rate transmission for point-to-point links.

The fabrication of the TWT is in progress.

\section{Acknowledgements}

This work has been supported by EPSRC grant $\mathrm{EP} / \mathrm{P} 015883 / 1$ in the framework of the CHIST-ERA project TERALINKS, "TERAhertz high power LINKS using photonic devices, tube amplifiers and Smart antennas" and partially supported by ULTRAWAVE project that has received funding from the European Union's Horizon 2020 research and innovation program under grant agreement no 762119.

\section{References}

[1] G Ducournau, P Szriftgiser, D Bacquet, A Beck, T Akalin, E Peytavit, M Zaknoune and J F Lampin 2010 Optically power supplied Gbit/s wireless hotspot using $1.55 \mu \mathrm{m} \mathrm{THz} \mathrm{photomixer}$ and heterodyne detection at $200 \mathrm{GHz}$ Electron. Lett. 46(19) 1349

[2] M Tonouchi 2007 Cutting-edge terahertz technology Nat. Photon. 1(2) 97 
[3] E Peytavit, P Latzel, F Pavanello, G Ducournau and J F Lampin 2013 CW source based on photomixing with output power reaching $1.8 \mathrm{~mW}$ at 250 IEEE Electron Device Lett. 34(10) 1277

[4] S S Dhillon, M S Vitiello, E H Davies, A G Davies, M C Hoffmann, J Booske, C Paoloni, M Gensch, P Weightman, G P Williams and E Castro-Camus 2017 The 2017 terahertz science and technology roadmap J. Phys. D: Appl. Phys. 50043001

[5] T Nagatsuma, D Guillaume and Cyril C. Renaud 2016 Advances in terahertz communications accelerated by photonics Nat. Photon. 10(6) 371

[6] C Paoloni, F Magne, F André, J Willebois, Q Trung Le, X Begaud, G Ulisse, V Krozer, R Letizia, M Marilier, A Ramirez and R Zimmerman 2018 Transmission Hub and Terminals for Point to Multipoint W-band TWEETHER System Proc. European Conference on Networks and Communications pp 19

[7] T B Reed, M Rodwell, Z Griffith, P Rowell, A Young, M Urteaga and M A Field 2012 A $220 \mathrm{GHz}$ InP HBT Solid-State Power Amplifier MMIC with $90 \mathrm{~mW}$ POUT at $8.2 \mathrm{~dB}$ Compressed Gain Proc. IEEE Compound Semicond. Integr. Circuit Symp. (CSICS), pp 1-4

[8] J C Tucek, M A Basten, D A Gallagher and K.E. Kreischer 2012 $220 \mathrm{GHz}$ power amplifier development at Northrop Grumman Proc. IEEE $13^{\text {th }}$ Int. Vac. Electron. Conf. pp 553-554

[9] M A Basten, J C Tucek, D A Gallagher and K E Kreischer 2013 G-Band Power Module Development at Northrop Grumman Proc. IEEE 14th Int. Vac. Electron. Conf pp 1-2

[10] A Baig, D Gamzina, R Barchfeld, C Domier, L R Barnett and N C Luhmann $20120.22 \mathrm{THz}$ wideband sheet electron beam traveling wave tube amplifier: Cold test measurements and beam wave interaction analysis Phys. Plasmas 19(9) 093110

[11] C Paoloni, A Di Carlo, F Bouamrane, T Bouvet, A J Durand, M Kotiranta, V Krozer, S Megtert, M Mineo and V Zhurbenko 2013 Design and Realization Aspects of 1-THz Cascade Backward Wave Amplifier Based on Double Corrugated Waveguide IEEE Trans. Electron Devices 60(3) 1236

[12] D Gamzina, L G Himes, R Barchfeld, Y Zheng, B K Popovic, C Paoloni, E EunMi and N C Luhmann Jr. 2016 Nano-CNC Machining of sub-THz Vacuum Electron Devices IEEE Trans. Electron Devices 63(10) 4067

[13] D. Gamzina, H Li, L Himes, R Barchfeld, B Popovic, P Pan, R Letizia, M Mineo, J Feng, C Paoloni and N C Luhmann Jr. 2016 Nanoscale Surface Roughness Effects on $\mathrm{THz}$ Vacuum Electron Device Performance IEEE Trans. Electron Devices 15(1) 85

[14] C D Joye, J P Calame, M Garven and B Levush 2010 UV-LIGA microfabrication of $220 \mathrm{GHz}$ sheet beam amplifier gratings with SU-8 photoresist J. Micromech. and Microeng. 20 (12) 125016

[15] A Malekabadi and C Paoloni 2016 UV-LIGA microfabrication process for sub-terahertz waveguides utilizing multiple layered SU-8 photoresist J. Micromech. and Microeng 26(9) 095010

[16] M Mineo and C Paoloni 2010 Double-Corrugated Rectangular Waveguide Slow-Wave Structure for Terahertz Vacuum Devices IEEE Trans. Electron Devices 57(11) 3169

[17] C Paoloni and M Mineo 2014 Double Corrugated Waveguide for G-Band Traveling Wave Tubes IEEE Trans. Electron Devices 61(2) 4259

[18] C Paoloni, M Mineo, M Henry and P G Huggard 2015 Double corrugated waveguide for ka-band traveling wave tube IEEE Trans. Electron Devices 62(11) 385

[19] X Li , X Huang, S Mathisen, R Letizia and C Paoloni 2018 Design of 71-76 GHz Double-Corrugated Waveguide Traveling-Wave Tube for Satellite Downlink IEEE Trans. Electron Devices 65(6) 2195

[20] Mathisen, S., Basu, R., Billa, L.R., Gates, J., Rennison, N.P., Letizia, R., Paoloni, C., "Low Cost Fabrication for W-band Slow Wave Structures for Wireless Communication Travelling Wave Tubes", Proc. IEEE 19th Int. Vac. Electron. Conf. 2018.

[21] C Paoloni, D Gamzina, L Himes, B Popovic, R Barchfeld, L Yue, Y Zheng, X Tang, Y Tang, P Pan, H Li, R Letizia, M
Mineo, J Feng and N C Luhmann, Jr. 2016 THz BackwardWave Oscillators for Plasma Diagnostic in Nuclear Fusion," IEEE Trans. on Plasma Sci. 44(4) 369

[22] CST STUDIO SUITE ${ }^{\mathrm{TM}}, \quad$ CST AG, Germany [Online] http://www.cst.com

[23] A. S. Gilmour, Jr., Principles of Traveling-Wave Tubes. Boston, MA: Artech House, 1994. 